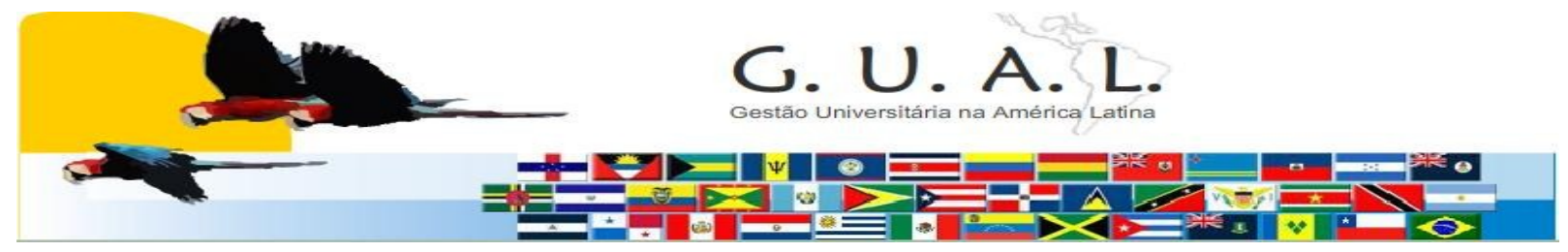

ISSN 1983-4535

\title{
ADMINISTRACIÓN DE LA EDUCACIÓN SUSTENTADA EN LA TEORÍA DE LA COMPLEJIDAD: UN ENFOQUE EMERGENTE EN LA SOCIEDAD DE LA INFORMACIÓN
}

\author{
Guiselle María Garbanzo Vargas, Mestre \\ Universidad de Costa Rica \\ gmgarban@gmail.com
}

\begin{abstract}
RESÚMEN
La autora ofrece un replanteamiento de la administración de la educación desde la teoría de la complejidad en el contexto de la sociedad de la información. Se considera vital el repensar la administración de la educación como disciplina y estrategia para alcanzar que las organizaciones educativas en la postmodernidad logren los niveles de competitividad que requieren las demandas de los nuevos tiempos. Se considera impostergable un abordaje y enfoque desde la interdisciplinaridad y la complejidad como visiones epistemológicas en las organizaciones educativas en un mundo globalizado. Los profesionales en el campo de la administración de la educación ante la emergente sociedad de la información requieren un replanteamiento de su rol dentro de las organizaciones educativas, capaces de desarrollar habilidades y competencias para gestar bajo un mundo complejo e incierto, dimensiones a considerar en el presente artículo.
\end{abstract}

Palabras claves: Administración de la educación. Teoría de la complejidad. Sociedad de la información y el conocimiento., Gestión de la educación. 


\section{CONTEXTUALIZACIÓN}

La sociedad del conocimiento, que ha caracterizado las tendencias globales en las que se dinamiza el mundo hoy, presenta consigo el renacer de una serie de variables implícitas en ella como lo son las tecnologías de la información y la comunicación, la globalización, énfasis en los servicios, aceleración del cambio, la imprevisibilidad, la inestabilidad y la incertidumbre. Todas ellas sumamente complejas entre sí e interrelacionadas en forma compleja; cuya interacción ofrece un repensar e interpretación del orden social, especialmente en su formas de organización y gestión, bajo el cual actúan las estructuras sociales.

El nuevo orden mundial ha traído un repensar fenomenológico de todos sus componentes, incluyendo nuevos paradigmas organizacionales, de gestión y del mismo sujeto, donde las organizaciones se transforman en verdaderos ambientes de cambio, en las que la innovación, la creatividad, la planeación, la adaptación y la flexibilidad son una constante.

La sociedad de la información y el conocimiento, que emerge como una especie de metamorfosis social, pasó de una sociedad industrial, en la que su principal factor de producción era el capital, hoy ha tomado el lugar el uso intensivo y competitivo del conocimiento. Experimentó una transición de una estructura vertical a una sociedad interconectada en redes, y desplazando el control como medio de resultados organizacionales, para darle lugar a la innovación como eje de eficiencia y eficacia organizacional. Romero (2000).

De esta manera las organizaciones educativas, insertadas como células vivas en la sociedad de la información, experimentan una transición, en las que han dejando patrones tradicionales para dar paso a organizaciones inteligentes, capaces de aprender por sí mismas, estructuras interconectadas en redes, gestores que acceden al conocimiento y con habilidad para interpretarlo y aplicarlo en forma inmediata al servicio de su comunidad de aprendizaje, según las aceleradas demandas del contexto.

\section{LA EDUCACIÓN EN LA SOCIEDAD DE LA INFORMACIÓN}

La educación es un instrumento indispensable, como camino hacia la paz, justicia social y libertad. Posee un papel esencial en el desarrollo continuo de la persona y de la sociedad como un todo. En busca de un desarrollo humano más armonioso, inclusivo y justo 
Delours (1996). La educación trabaja facilitando dos componentes fundamentales a saber, el desarrollo cognitivo y espiritual de la persona, como medios para alcanzar la plenitud como individuo planetario consigo mismo, y con los demás.

Por ser la educación un proceso para el desarrollo de la persona en forma integral y continuo, por sí misma es altamente compleja para que una sola disciplina la aborde, su comprensión ocupa de otras disciplinas, requiere de la transdisciplinaridad e interdisciplinaridad. Su proceso es más que una trasmisión de conocimientos, al buscar la educación el desarrollo del ser humano en sus diferentes etapas, desde una visión holística, hace de la administración de la educación un reto interdisciplilnario sustentado en la complejidad en forma constante para su operacionalización.

El nuevo orden social, ha replanteado las formas de enseñanza, la transmisión del conocimiento, ya no es exclusivo de las organizaciones educativas, el conocimiento está disponible mediantes diferentes redes en las cuales se interconecta la aldea global. Incluso el modelo de familia, también ha sufrido cambios en el siglo XXI, las organizaciones educativas tienen como reto gestar en un nuevo paradigma de socialización, donde la diversidad y la democratización de la familia son claves. Al respecto considera Romero (2005; pág. 139), que "la escuela tiene entonces por delante el conflicto de resolver una socialización en la diversidad".

En la sociedad del conocimiento el no acceso al conocimiento desde el punto de vista del proceso educativo, es considerado exclusión social, la cual puede incidir en la educación como el no acceso del todo a la educación, el abandono producto de la repitencia y el no poder garantizar calidad en los aprendizajes. (Romero; 2005). Estas dimensiones educativas son retos subyacentes de la administración de la educación, para que todos tengan la oportunidad de aprender y concretar su proceso educativo, lo que le implica a la gestión de la educación definir nuevas formas de aprendizaje en una educación inclusiva, donde en palabras de Delour, (1996) refiriéndose a los cuatro pilares de la educación considera que se debe aprender a aprender, aprender a conocer, aprender a hacer, aprender a vivir juntos y aprender a ser.

Estos retos subyacentes de la administración de la educación se ven insertados en sus organizaciones, las cuales en forma articulada emergen con nuevas formas de repensarse a sí

Rev. GUAL., Florianópolis, v.4, n. 1, p.27-43, jan/abr. 2011 
mismas, entre sí y entre los sistemas en los que son partes, como unidades sociales del complejo mundo social que caracteriza la postmodernidad.

\subsection{Organizaciones educativas en la sociedad emergente}

En el complejo contexto actual, las organizaciones que sobresalen no son las que poseen más recursos materiales y financieros. Hoy las organizaciones más exitosas son aquellas dotadas de conocimiento, capaces de seleccionarlo según los intereses de la organización e interpretarlo y ponerlo en práctica. El conocimiento en la actualidad es la principal herramienta con que las nuevas estructuras sociales compiten entre sí, para asegurar su posicionamiento de manera competitiva según las demandas de los tiempos.

El éxito de las organizaciones educativas va a depender de la capacidad de sus administradores de interpretar la realidad externa, reconocer las oportunidades que se presentan y sus transformaciones. Además, de la habilidad para responder en forma previsible e inteligente con los cambios y estrategias necesarias para que el proceso educativo, que es su razón de ser experimente las mínimas dificultades en su operacionalización, aportando a la formación del ser humano, como una persona, plena e integral en todas sus dimensiones cognitivas; aportando en el desarrollo de la capacidad cognitiva humana, la cual es generativa, plasmando lo moral y lo cultural, lo ético y reflexivo, colaborando con la construcción de una sociedad más justa e inclusiva para todos.

En el mundo globalizado las organizaciones han experimentado una especie de metamorfosis, que actúan en un amplio y complejo horizonte colmado de oportunidades, amenazas, riesgos y continencias, permitiendo renacer nuevas tendencias de la administración moderna Chiavenato (2002). Dentro de estos enfoques de la administración emergentes en la sociedad del conocimiento, se propone en este ensayo, la visión fundamentada de la administración en la teoría de la complejidad; la cual tiene como objetivo explicar desde la complejidad los procesos de gestión en los nuevos escenarios globales caracterizados por la incertidumbre, el caos y la complejidad, como lo expone Beck (1999), estamos ante la sociedad del riesgo. Al considerarse la sociedad del conocimiento como la sociedad del riesgo, de la complejidad, de la incertidumbre, este estado complejo que abate a las organizaciones educativas requiere de una gestión en particular, que responda a esta particularidad característica de la postmodernidad. 


\section{ADMINISTRACIÓN DE LA EDUCACIÓN SUSTENTADA EN LA TEORÍA DE LA COMPLEJIDAD: UN ENFOQUE EMERGENTE EN LA SOCIEDAD DE LA INFORMACIÓN}

\subsection{Administración de la Educación sustentada en la Teoría de la Complejidad}

La mayoría de los problemas que afligen a las organizaciones hoy tienen su causa en la capacidad de gestión, en la forma en que son administradas; estas requieren de administraciones ágiles y flexibles, de manera que se mantengan competitivas dentro del dinamismo, complejo e incierto ambiente en que les ha tocado desarrollarse.

Estas nuevas tendencias de la postmodernidad traen una nueva forma de reinvención de la administración de la educación, como única forma de desarrollar las organizaciones educativas en forma ágil, pertinente y competitiva, sobre la incertidumbre e inestabilidad que caracteriza este orden mundial. Las organizaciones de la educación ante estas características se encuentran repensándose a sí mismas, sobre todo, emerge una nueva forma de gestión, centrada en la complejidad e incertidumbre, donde la certeza y el riesgo dan paso a lo inesperado e imprevisible, es un reto de la administración de la educación en la postmodernidad; trabajar con estas características.

La incertidumbre se da cuando los ambientes cambian con rapidez, por una serie de variables que operan en forma constante en líneas imprevisibles, la administración, no posee la información necesaria sobre los eventos que hacen cambiar con rapidez y se desconoce la tendencia sobre la cual la organización continuará Chiavenato (2002). Al respecto, es importante revisar si ¿Está preparada la administración de la educación para asumir tan compleja tarea? ¿Puede seguir actuando la administración de la educación con un enfoque tradicional ante las demandas de la postmodernidad, caracterizado por un ambiente renovado, que cambia con rapidez, complejo, dinámico e incierto?

La administración de la educación en el increíble ritmo de la aceleración, que caracteriza los nuevos tiempos, requiere nunca como hoy de la eficiencia y la eficacia de las habilidades de los administradores y administradoras para concretar el proyecto de organización educativa. Los profesionales encargados de la administración tienen bajo su responsabilidad el desempeño de los integrantes de sus organizaciones, lo que determina los resultados obtenidos por la administración.

La ciencia de la administración de la educación, radica en la capacidad de conducir las acciones de los miembros integrantes en busca del logro de los objetivos colectivos, por los cuales la organización educativa trabaja. Es la capacidad de planear, dirigir personas, organizar, controlar recursos y gestionar Chiavenato (2002).

Rev. GUAL., Florianópolis, v.4, n. 1, p.27-43, jan/abr. 2011 


\section{ADMINISTRACIÓN DE LA EDUCACIÓN SUSTENTADA EN LA TEORÍA DE LA COMPLEJIDAD: UN ENFOQUE EMERGENTE EN LA SOCIEDAD DE LA INFORMACIÓN}

En manos de la administración está el servir hacia el desarrollo óptimo de sus ideales, imprimir liderazgo y disposición sobre la disposición y uso de recursos. La administración como ciencia social debe demostrar la capacidad de trabajar con personas, dirigir sus acciones, creación de un ambiente idóneo en busca de la consecución de los objetivos organizacionales. La administración logra que otras personas realicen lo que tienen que hacer por el bien organizacional. Le corresponde a la administración de la educación actuar en ambientes variables, por su propia naturaleza opera en la complejidad, en la incertidumbre, trabaja para el mañana, en el desarrollo de las personas que la sociedad requiere a largo plazo; condición que de por sí es ambigua y variable.

El talento por ninguna razón debe desaprovecharse ni otro tipo de recursos; todos ellos sumamente costosos. Tampoco puede desaprovecharse el conocimiento disponible, este es otro recurso que hoy las organizaciones educativas no pueden darse el lujo de malgastarlo, el conocimiento en las organizaciones de la sociedad de la información es el principal recurso que les permitirá la consecución de sus objetivos. Una inadecuada utilización del mismo puede sacar de la competitividad a cualquier organización educativa. No en vano entre las emergentes funciones de la administración de las organizaciones educativas contemporáneas, se considera la gestión como la aplicación del conocimiento existente para producir resultados; por consiguiente, un gestor de la educación, que ocupe un cargo directivo “... es responsable de la aplicación y rendimiento del saber [en la organización]" (Drucker, 1999, p. 61). Además, de la gestión, son consideradas la planificación desde la complejidad, el liderazgo y la investigación; todas ellas requieren de la gestión del conocimiento para su ejecución.

Los nuevos albores de la sociedad ha traído consigo nuevos problemas, nuevas formas de interacción, nuevos enfoques epistemológicos dialécticos, es decir nuevos saberes, es una revolución en las formas de pensamiento, pasando de una pensamiento plano, disciplinar a uno transdisciplinar, multidisciplinar en un marco de complejidad. Es una revolución de la concepción del hombre, sus modos de concebir y producir el conocimiento y la misma construcción de la ciencia como hasta hoy se ha concebido, es un nuevo paradigma de construir y producir el conocimiento y concebir el hombre. "Uno de los cambios sustanciales que dicha revolución trae consigo es la modificación del lugar del conocimiento científico en el sistema del saber humano, lo que conduce a la elaboración de un saber nuevo, que avanza 
de la mano de las soluciones teóricas innovadoras tales como enfoque de la Complejidad" Aguerrondo (2007; pág. 13).

El enfoque de la complejidad plantea la superación de la concepción clásica de la racionalidad; sus ideas trascienden hacia un ideal complejo. El enfoque de la complejidad concibe el mundo como una interacción de sistemas dinámicos, donde sus relaciones entre sí mismos y entre los sistemas con el entorno, adquieren un gran significado en la comprensión de estas complejas estructuras, donde cada una de sus partes interactúa con los componentes estructurales en los que actúa la misma sociedad. En este enfoque la diversidad es una variable más, no menos importante que la complejidad misma subyacente en las nuevas formas de concebir el mundo. En este contexto emerge una revolución científica en un mundo simplemente caracterizado por la complejidad.

La teoría de la Complejidad además, de traer consigo un cambio profundo en las formas de comprender las interacciones como un todo, presenta como es de esperarse una nueva forma de comprender la relación subyacente entre la persona y el mundo, una nueva forma de sociedad, es una nueva cosmovisión que ha revolucionado las estructuras hasta entonces aceptadas como válidas, conduciendo a un nuevo y complejo orden social. Maldonado (2003), llama la atención en el sentido de que la teoría de la Complejidad se mueve entre la complejidad como ciencia, como método de pensamiento y la complejidad como cosmovisión. La primera se refiere a una dinámica que supera lo no lineal en la complejidad de los sistemas. La segunda se refiere a un método de pensamiento que venga a superar las dicotomías simplistas de los métodos disciplinares por un aprendizaje del pensamiento en forma relacional. Finalmente la complejidad como cosmovisión invita a la construcción de una nueva forma de mirar y comprender el mundo y el saber, donde el reduccionismo es superado por una concepción holística.

La teoría de la Complejidad surge como un proceso natural que la evolución social en su ritmo natural ha hecho emerger, producto de sus propias conexiones, aciertos y desaciertos en forma racional o no, en forma planificada o no. Estas interacciones entre sí mismas son complejas, resultan aún más inciertos entre sí. Esta complejidad permea todas las células sociales, donde las organizaciones como unidades conectadas con un todo, les corresponde insertarse, y lo hacen en forma natural, no es planificado el pasar a formar parte de esta complejidad, simplemente son parte de este entorno. Ante este nuevo orden social, las 
organizaciones educativas requieren de nuevos marcos conceptuales de administrar para hacerle frente a las decisiones requeridas que permitan liderar y gestar a las alturas de los tiempos. De esta forma, se encuentra la administración de la educación ante la gestión del caos, de la incertidumbre, de la complejidad, es un replanteamiento epistemológico de hasta ahora su forma de administrar. Se está trascendiendo a una administración de la educación basada en la teoría de la Complejidad, es un nuevo enfoque emergente de la sociedad de la información.

Administrar en este contexto, en un orden social complejo, con organizaciones que no escapan de esta incertidumbre, del caos característico del nuevo orden social, conlleva a la administración de la educación a un nuevo enfoque de gestión, sustentado en la teoría de la complejidad, es repensar su forma de gestión hasta el momento. Es trascender de un una forma tradicional, lineal de gestión, para trascender en el marco del siglo XXI, a una teoría de la administración de la educación relacional, que supere las dicotomías de formas de administrar aislados, lineales para pasar al mundo de las interconexiones y las relaciones.

La administración de la educación sustentada en la teoría de la complejidad implica un nuevo pensamiento, nuevas competencias que emergen en forma interconectadas, es una nueva cosmovisión de la administración de la educación, donde se supere el reduccionismo en términos de consideraciones dialécticas, epistemológicas de manera holística, es gestar la complejidad.

Para poder atender los desafíos que las nuevas posturas epistemológicas traen en el marco de la incertidumbre, característica del nuevo orden social imperante en el siglo XXI, Aguerrondo (2007), citando a Courtney considera que en la auténtica ambigüedad actúan una serie de dimensiones de la incertidumbre imposibles de prever, es difícil identificar los variados escenarios y consecuencias que la complejidad de estas organizaciones, razón por la cual la teoría de la administración fundamentada en la complejidad surge como un paradigma que conlleva a repensar la gestión de la educación.

Este nuevo enfoque de la administración de la educación fundamentado en la teoría de la complejidad, conlleva al replanteamiento de distintas variables tales como:

- la planificación como estrategia de gestar la incertidumbre;

- capacidad de interpretar la realidad en forma acertada; 
- capacidad de gestión en ambientes complejos, turbulentos e inciertos;

- superación de modelos tradicionales de gestión;

- el surgimiento de una nueva conciencia organizacional;

- administración con pensamiento dialéctico y estratégico.

\subsection{La planificación como estrategia de gestar la incertidumbre}

Las organizaciones de hoy operan sobre ambientes inestables, es una condición necesaria en este contexto que la administración de la educación haga uso de forma impostergable de la planificación en forma responsable; ofreciéndole conocimiento acertado de la realidad bajo la cual actúan, redireccionando así el rumbo de las organizaciones educativas.

La planificación aplicada en las organizaciones educativas en forma apropiada es una herramienta con que cuentan los administradores de la educación para tomar decisiones acertadas, ya que la construcción de la planificación está sustentada en el conocimiento, el cual además de permitir una lectura social acertada sobre sus retos y desafíos, ofrece además, del conocimiento condiciones para dirigir y conducir la realidad. Aguerrondo (2007). Es la planificación educativa una estrategia para ejecutar los cambios que la educación requiere según las exigencias de los tiempos. En la actual era de la incertidumbre en la que se debaten las organizaciones educativas, la planificación de la educación es un medio para lograr las reformas estructurales que la educación requiere.

\subsection{Capacidad de interpretar la realidad en forma acertada}

Las organizaciones educativas de la postmodernidad requieren de parte de sus gestores la capacidad de aplicar el conocimiento disponible para la transformación estructural necesaria. (Duker; 1999). El conocimiento disponible va más allá del recibido en la formación académica, es una constante en el desempeño, es la búsqueda, capacidad de discernir, interpretar y aplicar el conocimiento en su desempeño, es una competencia impostergable de los gestores de la educación, que toma mayor protagonismo en la sociedad de la información. El reto de hoy no está en el acceso del conocimiento, ya que este gracias las tecnologías de la información (TICS), se encuentra disponible sin discriminación, el desafío radica en el uso 
inteligente que se le dé, y en la capacidad de ponerlo en práctica al servicio del colectivo común Castells (2003).

El conocimiento es necesario para poder dirigir la organización con plena comprensión de la realidad, puesto que la construcción del conocimiento se sustenta en el saber. Aguerrondo (2007). Es condición de quienes dirigen la educación, poseer conocimiento acertado de la realidad. La comprensión del mundo en forma interrelacionada, no lineal es la que se necesita para comprender el agitado mundo por la cual se debate hoy la educación desde sus diferentes espacios organizacionales.

Al estar el conocimiento en constante cambio, los gestores de la educación requieren habilidades de autoaprendizaje permanentes, las cuales son hoy una condición para que la educación alcance sus objetivos y pueda la sociedad competir con las demandas globales. Un administrador de la educación con una actitud constante para acceder al conocimiento que se ofrece al servicio de la comunidad globalizada, y además, posea las competencias profesionales para interpretarlo y aplicarlo hacia el logro de los objetivos de la educación, tiene altas posibilidades de encausar su organización por el camino correcto, según las exigencias económicas y éticas de la sociedad globalizada.

La capacidad de interpretar la realidad finalmente es comprender la incertidumbre de la realidad. Es poseer las destrezas y competencias para poder tener la capacidad de interpretar donde está la realidad, y es el acto congnitivo el que ofrece al capacidad de llegar a un acercamiento pertinente de lo real, y este es construido mediante la constancia de la incertidumbre, que le permite a las personas cuestionarse constantemente la supuesta realidad.

Finalmente, la construcción de conocimiento válido requiere la capacidad de construir e interpretar el conocimiento en forma articulada y no segmentada de la realidad, producto de los múltiples campos disciplinares, en la que prevalezca la percepción de la complejidad como un todo, facilitando una adecuada interpretación de la realidad. La realidad no es otra que nuestra interpretación de la realidad (Morán, 2000).

\subsection{Capacidad de gestión en ambientes complejos, turbulentos e inciertos}

La incertidumbre siempre ha existido en la historia de la humanidad, lo que ha traído consigo la sociedad postmoderna es la intensificación con que se da este nivel de incertidumbre debido al marcado mundo dinámico y acelerado de sus interconexiones, lo que 
incrementa el caos con respecto a la capacidad de predecir lo impredecible. La historia avanza, no en forma lineal, lo hace mediante una serie de desviaciones o acontecimientos externos, que de una u otra forma vienen a desarrollar y transformar el sistema. Estas transformaciones representan morfogénesis, promotoras de un nuevo orden, es una metamorfosis social que opera en sus distintas organizaciones, y no tienen otra forma de evolucionar sino mediante nuevos replanteamientos.

La historia ha sido y será por siempre expuesta a períodos de turbulencias, de riesgos, donde producto de estos estados es que han surgido nuevas formas de pensamiento y de orden social en consecuencia.

Al traer la sociedad de la información de forma implícita un nuevo paradigma de gestión de la educación, un modelo sustentado en la complejidad, emergen nuevas competencias del gestor de la educación, como lo es la impostergable capacidad de desempeñarse en la incertidumbre y el caos que caracteriza la sociedad emergente. En este nuevo modelo de gestión no hay espacio para el separación entre la razón y la decisión, es inaceptable el aislamiento entre práctica y la teoría, entre los decisiones y la capacidad de gestión, entre el desconocimiento y el saber. La teoría y la práctica representan un binomio dependiente entre sí.

Se le exige a los gestores de la educación respuestas inmediatas, cambios acelerados, innovaciones constantes, replanteamiento de objetivos según lo emergente. No es un espacio para respuestas pausadas. Todo ello hace que este profesional desarrolle competencias para operar sobre organizaciones en redes y comunidades interconectadas en forma virtual, que le facilite desempeñarse de manera dinámica y acertada.

La capacidad de gestión en ambientes complejos, turbulentos e inciertos es gestar siendo consciente de la complejidad de las organizaciones educativas, del riesgo persistente, de lo imprevisto, del caos, de lo imprevisto, de las transformaciones, del cambio de dirección que también es una constante propia de las organizaciones de la era de la complejidad, se debe partir de que la certeza no es una variable a considerar como sí lo es la complejidad.

Las organizaciones educativas se mueven ante complejas condiciones ineludibles entre la decisión, la economía, lo social, la decisión; todas ellas operan en forma compleja entre sí y en forma articulada, donde la incertidumbre es la única certeza de su escenario organizacional, en la que la lógica positivista de la causa y efecto no necesariamente es una 
constante. Le corresponde a la gestión de la educación hacer previsible lo imprevisible. Es repensar la administración de la educación en el marco de las nuevas perspectivas.

La precaución y la estrategia son consideradas formas de enfrentar la incertidumbre. La estrategia significa desarrollar un programa, que toma en cuenta las certezas y las incertidumbres, así como las probabilidades y las improbabilidades Morán (2000). De esta manera es posible actuar sobre la organización, y para ello se debe partir del principio de que la complejidad se puede gestar pero no eliminar. Así las cosas, la gestión de la educación debe enfocarse para administrar la incertidumbre. Es la capacidad de gestar para que lo improbable se haga probable.

\subsection{Superación de modelos tradicionales de gestión}

Hoy más que nunca la educación es concebida como un medio estratégico en el futuro de la humanidad, donde la actual coyuntura en la que se estremecen los nuevos paradigmas económicos y sociales, replantean nuevos modelos de su forma de gestión, dejando atrás los modelos tradicionales cuyas prácticas de gestión no son compatibles con las exigencias de la actual sociedad.

Las organizaciones educativas para ser competitivas deben superar sus modelos tradicionales, en los que los planes una vez aprobados cumplen un requisito formal y carecen de práctica. Modelos donde la estructura es vertical, dando paso a estructuras horizontales interconectadas entre redes, entre ellas mismas y entre los componentes del sistema y la comunidad globalizada. Dejando atrás una gestión enfocada a las rutinas administrativas, para dar paso a un modelo de gestión centrado en lo pedagógico de forma interdisciplinario y multidisciplinaria. La gestión lineal no es una opción para las organizaciones de la postmodernidad, por sus propias características la sociedad del conocimiento aboca por prácticas de gestión dinámicas, innovadoras, centradas en lo estratégico con una visión inteligente del entorno.

La gestión de la educación tradicional, pareciera que se mueve entre la imposibilidad de capacidad de gestión de lo proyectado, marcando una evidente distancia entre la realidad, donde opera una evidente incapacidad de adaptarse a los cambios del entorno, el cual cada día es más dinámico, y requiere de la administración de la educación una intervención efectiva de sus decisiones, que se mueva en forma fluida en la complejidad subyacente. Es la 
capacidad de gestionar con procesos de planificación la previsión o el cálculo de situaciones en un escenario social de incertidumbre y turbulencia. Matus (1987) citado por Aguerrondo (2007).

\subsection{EI surgimiento de una nueva conciencia organizacional}

Esta surge en el momento que las personas han tenido que enfrentarse a la incertidumbre. (Morán, 2000). Las organizaciones se abaten entre la ambivalencia, es así que las organizaciones de la educación debe volver su gestión hacia la complejidad y la incertidumbre. Lo que asume valor y es relevante es la capacidad de la administración de la educación de reconocer la complejidad y la incertidumbre de la realidad en la que operan las organizaciones educativas en el cumplimiento del proceso educativo. La realidad, no necesariamente es perceptible desde los distintos saberes. Las organizaciones no existen en el vacio actúan en ambientes sumamente competitivos y dinámicos

\subsection{Administración con pensamiento dialéctico y estratégico}

Requiere de gestores de la educación con una mentalidad abierta, ágil, flexible e innovadora, que les permita desarrollar un pensamiento estratégico y dialéctico. Implica la capacidad de reconocer la complejidad del entorno social como un todo y en forma profunda, es más que reconocer sus componentes, es identificar su profundidad y precisar la interpretación epistemológica también. Una adecuada lectura dialéctica de la organización educativa facilita los procesos de planificación y decisión. Implica desde la administración la capacidad de rastrear los cambios y las transformaciones sociales y ofrecer respuestas en forma adecuada $\mathrm{y}$ en términos de plazos sensatos. Es una visión interdisciplinaria y multidiscplinaria.

Finalmente, son evidentes las megatendencias de la administración de la educación sustentada en la teoría de la complejidad, como un enfoque emergente, donde la administración de la educación de corte tradicional se aleja para darle lugar a un nuevo enfoque emergente sustentado en la teoría de la complejidad como respuesta los múltiples desafíos que la nueva sociedad de la información y el conocimiento ha traído consigo impactando en todas las esferas sociales, y poniendo a los sistemas educativos en claros desafíos y abatiéndose en espacios de gestión turbulentos. 


\section{Quadro I}

Megatendencias de la administración de la educación en la complejidad del mundo moderno

\begin{tabular}{|c|c|}
\hline Enfoque tradicional & $\begin{array}{c}\text { Enfoque moderno sustentado en la } \\
\text { complejidad }\end{array}$ \\
\hline $\begin{array}{l}\text { Particularidad y estándares mínimos } \\
\text { aceptables. }\end{array}$ & Pluralismo y competitividad. \\
\hline Participación limitada. & Democratización. \\
\hline $\begin{array}{l}\text { Estructuras que no requieren } \\
\text { necesariamente de cambios transcedentales } \\
\text { para operar. }\end{array}$ & Innovación constante. \\
\hline Entorno social conservador. & $\begin{array}{l}\text { Necesidad de realizar en forma constante } \\
\text { lectura social de su entorno. }\end{array}$ \\
\hline Pensamiento lineal. & Pensamiento estratégico y sistémico. \\
\hline $\begin{array}{l}\text { Se desempeña más que todo en un plano } \\
\text { institucional. }\end{array}$ & Opera sobre redes interconectadas. \\
\hline Visión disciplinaria. & Visión interdisciplinaria y multidisciplinaria. \\
\hline $\begin{array}{l}\text { Herramientas mínimas y tradicionales para } \\
\text { operar. }\end{array}$ & Uso inteligente de las TICs \\
\hline Se responde a estándares tradicionales. & Habilidad para determinar prioridades. \\
\hline Resultados individualizados. & Resultados globales organizacionales. \\
\hline $\begin{array}{l}\text { El trabajo opera sobre enfoques } \\
\text { tradicionales, más que todo orientado del } \\
\text { pasado para el presente. }\end{array}$ & Orientación hacia el futuro. \\
\hline $\begin{array}{l}\text { Profesionales que operan bajo una visión } \\
\text { individualizada. }\end{array}$ & $\begin{array}{l}\text { Movilidad personal y profesional (capacidad de } \\
\text { comprender un mundo con diferentes culturas, } \\
\text { costumbres, ambientes etc) }\end{array}$ \\
\hline $\begin{array}{l}\text { Organizaciones que actúan en ambientes } \\
\text { estables. }\end{array}$ & $\begin{array}{l}\text { Las organizaciones no existen en el vacio } \\
\text { actúan en ambientes sumamente competitivos y } \\
\text { dinámicos. }\end{array}$ \\
\hline Identificación de amenazas y debilidades. & $\begin{array}{l}\text { No solo identificar las amenazas y debilidades } \\
\text { sino capacidad para neutralizarlas. }\end{array}$ \\
\hline
\end{tabular}

Elaboración propia: Garbanzo (2010).

\section{CONCLUSIONES}

Los principales problemas que enfrentan las organizaciones se deben a la capacidad de gestión, por lo general no operan con fuerza otras causas. Las organizaciones requieren de administraciones ágiles y flexibles, de manera que se mantengan competitivas en el dinámico y complejo ambiente en el que les corresponde operar. 


\section{ADMINISTRACIÓN DE LA EDUCACIÓN SUSTENTADA EN LA TEORÍA DE LA COMPLEJIDAD: UN ENFOQUE EMERGENTE EN LA SOCIEDAD DE LA INFORMACIÓN}

Las nuevas tendencias de la postmodernidad conllevan a una reinvención de la administración de la educación, como única forma de desarrollar las organizaciones educativas en forma ágil, pertinente y competitiva, sobre la incertidumbre e inestabilidad que caracteriza este orden mundial. Es así, que las organizaciones de la educación se encuentran repensándose a sí mismas, emerge una nueva forma de gestión, centrada en la complejidad.

Le corresponde a la administración de la educación actuar en ambientes variables, por su propia naturaleza opera en la complejidad, en la incertidumbre, trabaja para el mañana, en el desarrollo de las personas que la sociedad requiere a largo plazo; condición que de por sí es ambigua y variable.

El conocimiento en las organizaciones de la sociedad de la información es el principal recurso que les permitirá la consecución de sus objetivos. Una inadecuada utilización del mismo puede sacar de la competitividad a cualquier organización educativa. La gestión en las organizaciones educativas contemporáneas le corresponde aplicar el conocimiento existente para producir los resultados que la sociedad espera de ella, de manera que la educación logre altos niveles de competitividad según las demandas de los nueva era.

Los nuevos albores de la sociedad ha traído consigo nuevos problemas, nuevas formas de interacción, nuevos enfoques epistemológicos dialécticos y nuevos saberes. Es una revolución en las formas de pensamiento, ante lo cual la administración de la educación debe responder en forma transdisciplinaria y multidisciplinaria, capaz de trabajar en redes de manera interconectada en un marco de complejidad, de manera que supere la concepción clásica de la racionalidad; sus ideas trascienden hacia un ideal complejo. La complejidad como cosmovisión invita a la construcción de una nueva forma de mirar y comprender el mundo y el saber, donde el reduccionismo es superado por una concepción holística.

La sociedad de la información y la comunicación ha llevado a la administración de la educación ante la gestión del caos, de la incertidumbre, de la complejidad, es un replanteamiento epistemológico de hasta ahora su forma de administrar. Se está trascendiendo a una administración de la educación basada en la teoría de la Complejidad, es un nuevo enfoque emergente de la sociedad de la información.

La administración de la educación sustentada en la teoría de la complejidad implica un nuevo pensamiento, nuevas competencias que emergen en forma interconectadas, es una nueva cosmovisión de la administración de la educación, donde se supere el reduccionismo en

Rev. GUAL., Florianópolis, v.4, n. 1, p.27-43, jan/abr. 2011 
términos de consideraciones dialécticas, epistemológicas de manera holística, es gestar la complejidad.

Este nuevo enfoque de la administración de la educación fundamentado en la teoría de la complejidad, conlleva al replanteamiento de distintas variables tales como: La planificación como estrategia de gestar la incertidumbre, capacidad de interpretar la realidad en forma acertada, capacidad de gestión en ambientes complejos, turbulentos e inciertos, superación de modelos tradicionales de gestión, el surgimiento de una nueva conciencia organizacional, administración con pensamiento dialéctico y estratégico.

\section{BIBLIOGRAFIA}

AGUERRONDO, Inés. Racionalidades subyacentes en los modelos de planificación (educativa). In: X curso regional sobre formulación y administración e Políticas educativas. Buenos Aires, Argentina: IIPE/UNESCO, 2007.

BECK, U. (1999). Hijos de la libertad. Fondo de Cultura Económica. Buenos Aires, Argentina.

CHIAVENATO, Idalberto. Administración en los nuevos tiempos. Bogotá, Colombia: McGraw-Hill S.A, 2002.

DELOURS, J. La educación encierra un tesoro. Madrid, España: Unesco/Santillana, 1996.

DRUCKER, Peter. La sociedad poscapitalista. Buenos Aires: Sudamericana, 1999.

MALTODANO, C.E. Marco Teórico del trabajo en ciencia de la complejidad y siete tesis sobre la complejidad. In: Revista Colombiana de Filosofía de la Ciencia, Vol. 4, nº 8-9. Bogotá, Colombia: Universidad del Bosque, 2003.

MORÍN, Edgar. (2000). Los siete saberes necesarios a la educación del futuro. Caracas, Venezuela: IESALC/UNESCO, 2000.

ROMERO, Claudia. La Institución escolar en la sociedad del conocimiento. Conferencia. Universidad de San Andrés y la Fundación Lúminis, 2005. 


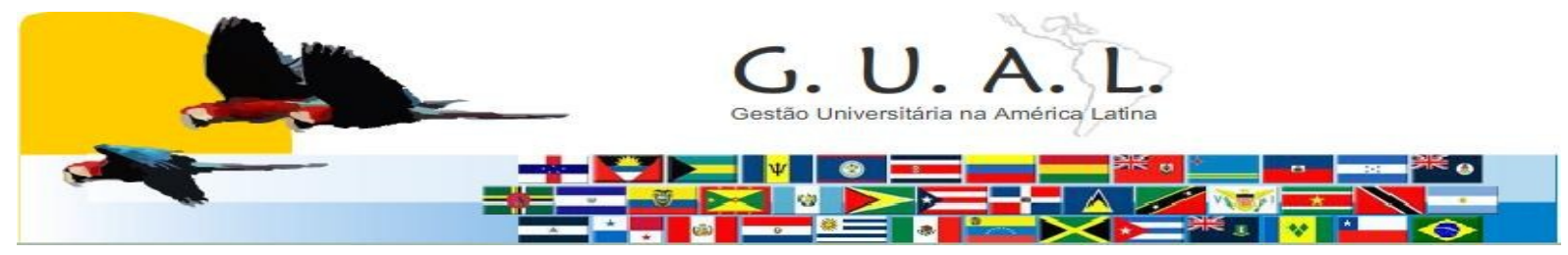

ISSN 1983-4535

\title{
ADMINISTRATION OF EDUCATION BASED ON THE THEORY OF COMPLEXITY: ANEMERGING APPROACH IN THE INFORMATION SOCIETY
}

\author{
Guiselle María Garbanzo Vargas, Master \\ University of Costa Rica \\ gmgarban@gmail.com
}

\begin{abstract}
The author offers a reassessment of the educational administration from the theory of complexity (point of view), in the society of information context. It is considered essential to rethink the educational administration as a discipline and strategy to make that educational organizations in post modernity reach the competitivity levels that new times demand. Besides, the author considers important to take another look to interdisciplinarity and complexity, as epistemological visions in educational organizations in a globalized world. Professionals in educational administration, require a reassessment of their roles on educational organizations, changes that make a professional able to develop abilities to manage education in a complex world, because of the emergent information society.
\end{abstract}

Keywords: Educational administration. Complexity theory. Information and knowledge society. Educational management. 